\title{
Transcriptional control of vertebrate neurogenesis by the proneural factor Ascl1
}

\section{Francisca F. Vasconcelos and Diogo S. Castro*}

Molecular Neurobiology, Instituto Gulbenkian de Ciência, Oeiras, Portugal

\section{Edited by:}

Marcos R. Costa, Federal University of Rio Grande do Norte, Brazil

\section{Reviewed by:}

Joao R. L. Menezes, Universidade

Federal do Rio de Janeiro, Brazil

Carol Schuurmans, University of

Calgary, Canada

*Correspondence:

Diogo S. Castro, Molecular

Neurobiology, Instituto Gulbenkian de Ciência, Rua da Quinta Grande 6,

P-2780-156 Oeiras, Portugal

e-mail:dscastro@igc.gulbenkian.pt
Proneural transcription factors (TFs) such as Ascl1 function as master regulators of neurogenesis in vertebrates, being both necessary and sufficient for the activation of a full program of neuronal differentiation. Novel insights into the dynamics of Ascl1 expression at the cellular level, combined with the progressive characterization of its transcriptional program, have expanded the classical view of Ascl1 as a differentiation factor in neurogenesis. These advances resulted in a new model, whereby Ascl1 promotes sequentially the proliferation and differentiation of neural/stem progenitor cells. The multiple activities of Ascl1 are associated with the activation of distinct direct targets at progressive stages along the neuronal lineage. How this temporal pattern is established is poorly understood. Two modes of Ascl1 expression recently described (oscillatory $v s$. sustained) are likely to be of importance, together with additional mechanistic determinants such as the chromatin landscape and other transcriptional pathways. Here we revise these latest findings, and discuss their implications to the gene regulatory functions of Ascl1 during neurogenesis.

Keywords: Ascl1/Mash1, neurogenesis, proneural gene, transcription, Notch signaling

\section{INTRODUCTION}

Neurogenesis in the developing mammalian brain is a highly complex process that requires neural progenitor cells to progress through a succession of distinct cellular states. These developmental steps have been particularly well defined in the embryonic telencephalon, where distinct types of progenitors have been identified during the neurogenesis period (Kriegstein and Alvarez-Buylla, 2009). Radial glial (RG) cells in the ventricular zone (VZ) have characteristic features of neural stem/progenitor cells, as they self-renew by asymmetric division and have the potential to differentiate into both neurons and glial cells (Götz and Huttner, 2005). Upon cell division, RG cells give rise to another RG cell and either a post-mitotic neuron, or an intermediate progenitor that can divide further to amplify the lineage (Haubensak et al., 2004; Miyata et al., 2004; Noctor et al., 2004; Pilz et al., 2013). These various progenitor types are differentially segregated between two germinal layers. Most RG cells divide at the apical surface of the VZ, while most intermediate progenitors divide more basally in the sub-ventricular zone (SVZ).

Proneural transcription factors (TFs) of the bHLH family including Ascl1 (also called Mash1) and members of the Neurogenin family are the main regulators of vertebrate neurogenesis. Both gain and loss-of-function analyses have shown they are both required and sufficient to induce a complete program of neuronal differentiation (Bertrand et al., 2002; Wilkinson et al., 2013). While genetic ablation of Ascll in mice results in neural developmental defects associated with reduced generation of neurons (Casarosa et al., 1999; Horton et al., 1999; Marin et al.,
2000), overexpression of this TF in neural progenitors induces cell-cycle exit and full neuronal differentiation and specification (Nakada et al., 2004; Castro et al., 2006; Berninger et al., 2007b; Geoffroy et al., 2009). In line with its master regulatory role in the neuronal lineage, recent studies have revealed the ability of Ascl1 to convert various non-neural somatic cells (e.g., fibroblasts) into induced neurons (Berninger et al., 2007a; Vierbuchen et al., 2010; Karow et al., 2012), renewing interest in understanding how this important TF works at the molecular level.

\section{PRONEURAL FACTORS AND THE NOTCH SIGNALING PATHWAY}

While driving neuronal differentiation, proneural factors also activate the Notch signaling pathway in neighboring progenitors, a process that is highly reminiscent of the lateral inhibition model proposed for Drosophila neurogenesis (Louvi and Artavanis-Tsakonas, 2006). Proneural factors directly activate the transcription of Notch ligands such as Dll1 (Castro et al., 2006; Henke et al., 2009), which interact with a transmembrane Notch receptor in neighboring cells. This event results in the cleavage and release of the Notch intracellular domain (NICD) from the cell membrane into the nucleus, where it forms a complex with the DNA-binding TF Rbpj and additional coactivators. Direct targets of this complex include the bHLH transcriptional repressors Hes1 and Hes5, which in turn bind to the promoters of proneural genes, repressing their expression and thereby inhibiting neuronal differentiation (Kageyama et al., 2005). Thus, proneural genes are both regulators and regulated by the Notch 
signaling pathway, a network that functions in parallel to the differentiation program to keep-even if only transiently-adjacent cells undifferentiated. Such lateral inhibition results in proneural factors being expressed in a "salt-and-pepper" pattern and prevents simultaneous differentiation of all progenitors, ensuring that an appropriate number is maintained during embryonic development.

\section{A REVISED VIEW OF LATERAL INHIBITION IN VERTEBRATES}

It is known that in a variety of cell types (e.g., fibroblasts), Hes1 expression levels regularly alternate over time due to its ability to behave as an intrinsic oscillator (Hirata et al., 2002; Masamizu et al., 2006; Kobayashi et al., 2009). Hes1 represses its own promoter in a feedback mechanism, which associated with short-lived Hes1 transcript and protein, results in autonomous oscillations of its expression with a $2-3 \mathrm{~h}$ period. It was recently shown that Hes1 also oscillates in neural progenitors (Shimojo et al., 2008). Because Hes1 and proneural factors display complementary patterns of expression, one possibility is that oscillation of Hes 1 results in the oscillation of proneural genes. This is indeed the case for both Neurog2 and Ascl1, as shown by a variety of approaches (Shimojo et al., 2008; Imayoshi et al., 2013). Most notably was the generation of transgenic mice bearing a bacterial artificial chromosome (BAC) containing the Ascl1 regulatory regions driving the expression of Ascll fused to either luciferase or green fluorescent protein (GFP), where the activity of the reporter monitors faithfully the expression of the endogenous Ascl1 protein (Imayoshi et al., 2013). Proneural proteins are direct activators of Dll1, resulting in its oscillation and mutual activation of Notch signaling in neighboring progenitors (Castro et al., 2006; Shimojo et al., 2008). There is evidence that Hes1 activity both promotes and inhibits the cell cycle and therefore its oscillation may be required for cell proliferation (Castella et al., 2000; Hartman et al., 2004; Sang et al., 2008). Oscillatory expression of TFs with lineage specification functions has also been observed in other systems, and the function of such oscillations is still a matter of debate. As opposed to steady-state mode, oscillatory expression may generate heterogeneity of response of an apparently homogeneous progenitor cell population to a given input signal. In addition, different inputs may affect different parameters of the oscillation (e.g., period, amplitude) and, hence, trigger different functional outcomes (Mengel et al., 2010; Pina et al., 2012; Sequerra et al., 2013).

In light of these recent findings, the "salt-and-pepper" expression pattern of proneural factors is perceived as a snapshot of a dynamic mode of expression. Proneural factors are therefore expressed in neural progenitors at different stages of differentiation, and not only in committed progenitors that will soon become post-mitotic, as was previously thought (Figure 1).

\section{OSCILLATORY VS. SUSTAINED MODE OF EXPRESSION}

Time-lapse imaging of individual neural progenitors in culture revealed that during neuronal induction, Ascl1 and Neurog2 switch from an oscillatory to a sustained mode of expression after the last cell division, followed by the expression of the neuronal marker doublecortin 6-8 h later (Shimojo et al., 2008;

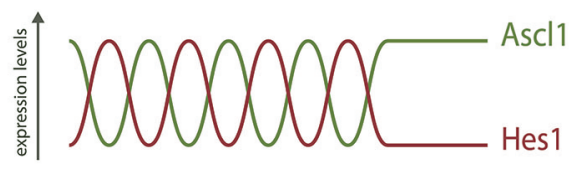

neural stem / progenitor cell

neuron

FIGURE 1 | Distinct modes of expression of Ascl1 in neural stem/progenitor cells. Hes1 and Ascl1 oscillate in neural progenitors with a 2-3 h period. The inverse correlation of expression of Hes1 and Ascl1 proteins suggest these TFs oscillate out-of-phase. At the onset of differentiation, Hes1 expression is extinguished and Ascl1 expression becomes sustained. The "salt-and-pepper" expression of Ascl1 in the neural tube is therefore the result of a dynamic pattern of expression, and Ascl1 expression is not necessarily an indicative of differentiation.

Imayoshi et al., 2013). This suggested the ability of proneural factors to trigger differentiation may require their expression to be sustained. Such a causal link was established upon the use of an optogenetic approach where Ascll expression is regulated by a light-switchable transactivator (Imayoshi et al., 2013). This system was introduced in an Ascl1 null background to investigate the consequence of different dynamics of Ascl1 expression induced by different pulses of light. An oscillatory mode with a $3 \mathrm{~h}$ periodicity increased proliferation, compensating the lower proliferation rate observed in Ascll null progenitors in culture. By contrast, sustained expression of Ascll for $6 \mathrm{~h}$ resulted in cell-cycle exit and neuronal differentiation. The same approach used in slice cultures of the dorsal telencephalon where Ascl1 is usually expressed at very low levels reached similar conclusions. What determines the transition to a sustained mode of Ascl1 expression remains an open question, but it was suggested that varying levels of NICD may play a role in this step (Imayoshi et al., 2013).

Overall, these findings explain why most evidence based on Ascl1 gain-of-function (sustained expression) points to a role in promoting differentiation (with concomitant cell cycle-exit) of progenitors (Nakada et al., 2004; Castro et al., 2006; Geoffroy et al., 2009). By contrast, knock-down of Ascl1 levels upon expression of sequence-specific shRNA decreased proliferation of neural progenitors in culture (Castro et al., 2011), while acute knock-out of Ascl1 in the ventral telencephalon caused premature cell-cycle withdrawal of both VZ and SVZ progenitors (Castro et al., 2011; Pilz et al., 2013), suggesting a role in maintaining proliferation in both RG cells and intermediate progenitors.

\section{CHARACTERIZATION OF AscI1 TARGET GENES}

Proneural factors function primarily as transcriptional activators, binding in heterodimeric complexes with bHLH E-proteins to the regulatory regions of their target genes (Bertrand et al., 2002). A major leap forward in our understanding of the molecular mechanisms underlying Ascl1 function has been the progressive characterization of its transcriptional program. The advent of genomic approaches based on chromatin immunoprecipitation allowed the characterization of a large number of genes directly controlled by Ascl1 in a neurogenesis context. Two studies have 
used chromatin immunoprecipitation followed by hybridization to DNA arrays (ChIP-chip), or massive parallel sequencing (ChIPseq), to characterize the Ascl1 transcriptional program in ventral telencephalon and dorsal spinal cord of the developing mouse embryo, respectively (Castro et al., 2011; Borromeo et al., 2014). A common theme of both studies was the diversity of biological functions of Ascl1 target genes, indicating Ascl1 directly controls various stages of neurogenesis, including neuronal differentiation, migration, axon guidance and synapse formation. In the ventral telencephalon, the region of the murine brain with the largest SVZ during the neurogenic period, the pro-proliferation activity of Ascll extends beyond the maintenance of Notch/Hes1 oscillations through activation of Dll1, and includes the activation of genes required for cell-cycle progression such as E2F1 and Foxm1 (Castro et al., 2011).

The Ascl1 targets are associated with progressive functions along neurogenesis and have distinct onsets of expression along the neuronal lineage in this brain region, as indicated by their expression patterns. The expression of the largest group mirrors that of Ascl1 itself in both germinal layers and includes genes expected to promote cell proliferation (e.g., E2F1), whereas that of a smaller but significant group of targets is restricted to the mantle zone (e.g., MAP2) (Figure 2).

All the above observations resulted in a model whereby Ascll sequentially promotes proliferation and differentiation of progenitors along the neuronal lineage, with the concomitant activation of partially distinct transcriptional programs. This reconciles the classical view of Ascll as a differentiation factor with the fact that this proneural factor is expressed mostly in cycling cells. The different kinetics of Ascll targets may result from the integration of various mechanistic determinants, including its two modes of expression as briefly discussed below.

\section{REGULATION OF AscI1 PROTEIN LEVELS AND TRANSCRIPTIONAL ACTIVITY}

One simple possibility is that increasing levels of Ascl1 activity, resulting from changes in the Ascll mode of expression (oscillatory vs. sustained), protein levels and/or its transcriptional function, will result in the sequential activation of promoters with an increasing threshold of response. Although not formally demonstrated, it is likely that some Ascll targets respond differently to the oscillatory or sustained expression of Ascl1. One possible model invokes the function of a feed-forward-loop (FFL), a motif highly enriched in transcriptional networks. A variant of such motif called coherent FFL (whereby one TF activates another TF, and both co-activate target genes) has been shown to allow for a discriminated response of the target gene triggered by transient or sustained input signals from the first TF (Shen-Orr et al., 2002; Mangan and Alon, 2003). Considering the large number of TFs found amongst Ascl1 targets, it would be of interest to investigate if any may establish with this proneural factor such a network motif, providing a mechanistic basis for differential activation of Ascll targets upon its two modes of expression.

Some observations suggest Ascll protein levels may also play a role at the onset of neuronal differentiation. Time-lapse

\section{Onset of expression of Ascl1 target genes}

A

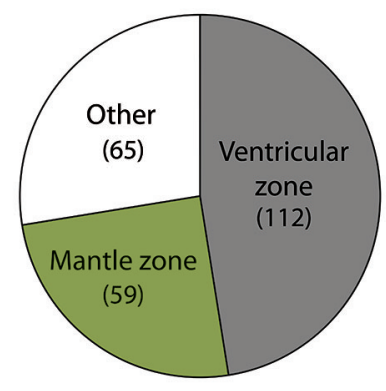

B
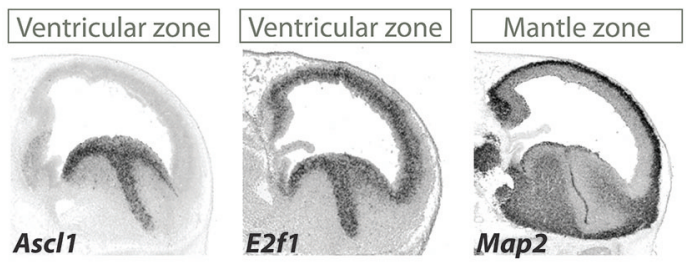

FIGURE 2 | Different patterns of expression of Ascl1 target suggest distinct kinetics of gene activation. (A) A large screening of expression patterns of Ascl1 direct targets in embryonic mouse ventral telencephalon revealed distinct patterns of expression (Castro et al., 2011). Three distinct groups can be identified. Genes in the largest set display an onset of expression at the VZ (which includes mostly neural stem cells), while in another the onset occurs at the mantle zone (here defined as including SVZ and all other outer layers, comprising intermediate progenitors and post-mitotic neurons). A third group includes genes with a uniform or more complex pattern of expression. Number of genes in each set is indicated in between brackets. (B) Examples of two Ascl1 direct targets with distinct patterns of expression revealed by in situ hybridization on sagittal sections of mouse telencephalon at E13.5 stage of embryonic development.

Expression of the proliferation gene E2f1 in VZ/SVZ mirrors that of Ascl1. By contrast, the neuronal differentiation gene MAP2 is expressed in post-mitotic neurons. Images are from Allen Developing Mouse Brain Atlas. ${ }^{1}$

imaging of Ascl1/luciferase expressing neural progenitors in culture revealed an increase in Ascl1 levels in 90\% of the cells that undergo asymmetrical (neurogenic) cell division, against $30 \%$ of the cells that undergo a proliferative symmetric division (Imayoshi et al., 2013). Thus, although not being strictly required or sufficient, an increase in Ascl1 protein levels before cell division does bias cells towards the neuronal fate.

A few signaling pathways have been implicated in the regulation of Ascll protein levels in different cellular contexts (Sriuranpong et al., 2002; Viñals et al., 2004; Oishi et al., 2009). In the most striking example, varying Ascl1 protein levels regulated by retinoic acid, result in the generation of different types of neurons at the $\mathrm{p} 3$ domain of hindbrain and spinal cord (Jacob et al., 2013). In all cases studied however, it is unclear if and how Ascll levels affect the activation of its target genes. Thus, while all these results indicate that various pathways converge to control Ascl1 protein levels, their relevance to the differential regulation of subsets of Ascll targets remains to be explored.

${ }^{1}$ Available at: http://developingmouse.brain-map.org 
Two recent studies provided evidence that Ascll function can be modulated by phosphorylation at multiple serine-proline sites. During cortical development, manipulating RAS/ERK signaling to abnormal high level induces a Neurog2 to Ascll switch of expression and modifies Ascll activity by direct phosphorylation. As a result, Ascl1 drives a glioblast-like differentiation program reminiscent of its function in oligodendrogenesis. Another work showed that Ascl1 phosphorylation is sensitive to levels of $\mathrm{Cdk} / \mathrm{Cdk}$ inhibitors, and diminishes its ability to drive primary neurogenesis in Xenopus embryos, providing a direct link between the cell-cycle machinery and regulation of neurogenesis (Ali et al., 2014). It is currently not known how phosphorylation impacts the neurogenic activity of Ascl1. Possible mechanisms include differential binding to DNA (as shown with Neurog2 phosphorylation) (Ali et al., 2011), or co-factor recruitment. Strikingly, phosphorylation affects the ability of Ascll to up-regulate the late/differentiation targets Myt1 and neural $\beta$-tubulin, while having little effect on Dll1 induction (Ali et al., 2014). Moreover, a differential effect is also observed on the ability of Ascll to transactivate the promoters of various target genes ( $\mathrm{Li}$ et al., 2014). Differential sensitivity of promoters to Ascll phosphorylation may thus be one important mechanism determining which targets Ascll regulates in proliferating vs. differentiating progenitors.

\section{IMPORTANCE OF CHROMATIN LANDSCAPE AND Ascl1 BINDING SITES}

Distinct thresholds of response to Ascl1 may result from differences in requirements for chromatin remodeling across its target genes. Very little is known however, on the impact that Ascll and proneural factors in general may have on the chromatin landscape when regulating gene transcription. Expression of a dominant negative form of Brg1, a catalytic component of SWI/SNF chromatin remodeling complex, blocks neuronal differentiation of P19 cells mediated by Neurog3 (Seo et al., 2005). In addition, it inhibits Neurog3 activation of the promoter of NeuroD2, the paradigm of a late/differentiation target of Neurogenins. In spite of this suggestive example, the importance of chromatin remodeling to the overall temporal patterning of the transcriptional program downstream of proneural factors remains to be investigated.

The chromatin landscape can contribute to restrict the accessibility of a TF to its target sites. A study of Ascl1 mediated neuronal reprogramming has recently shown that Ascl1 binds to its bona fide target sites when ectopically expressed in fibroblasts, even to those located within closed chromatin context, as defined by FAIRE-seq (Wapinski et al., 2013). The term "on target" pioneer factor was coined to indicate the ability of Ascll to recognize its cognate binding sites when ectopically expressed, as opposed to other TFs in iPS reprogramming (Soufi et al., 2012). Although the ability to bind nucleosomal DNA may argue against a dominant role of the chromatin structure in controlling Ascl1 function, it remains possible that Ascl1 accessibility to its target sites may be different in proliferating vs. differentiating progenitors.

The affinity of a TF binding site, determined by the DNA sequence, can dictate the kinetics of response of its direct targets.
A striking example of how such mechanism can establish the temporal pattern of a developmental program is the activation of pharyngeal genes by the forkhead factor PHA4 at different developmental stages in Caenorhabditis elegans (Gaudet and Mango, 2002). Binding site mutations result in abnormalities in the timing of target gene expression in vivo, according to the resulting affinity to PHA4 binding. Concerning bHLH TFs, and in addition to the two central bases of the E-box, which provide specificity to distinct factors, residues flanking the hexamer sequence contribute to modulate binding affinity (Blackwell and Weintraub, 1990; Fisher et al., 1993). A study investigating the regulation of the Dll1 gene by Ascl1 has shown that residues at each side and immediately adjacent to the CAGSTG E-box determine binding affinity of Ascl1 in vitro, and strength of response in transcriptional assays (Castro et al., 2006). Although a possibility, the contribution of varying affinities for its binding sites to the kinetics of Ascll targets remains to be investigated.

\section{FUNCTIONAL INTERACTIONS WITH OTHER TRANSCRIPTIONAL NETWORKS}

Transcriptional programs are at the intersection of multiple transcriptional networks. Thus, a comprehensive view of the dynamics of the Ascll program will require its integration within other transcriptional pathways operating in neural progenitors. Very few studies have so far identified functional interactions between Ascl1 and other TFs. The forkhead factor FOXO3 regulates neural stem cell maintenance and is required to preserve the neural stem cell pool in adult mice (Renault et al., 2009). Recently, it has been shown that FOXO3 inhibits Ascl1-induced neuronal differentiation in cultured neural progenitors and direct neuronal conversion in fibroblasts (Webb et al., 2013). Although the molecular basis for this antagonism is not yet clear, it is likely to make use of the large number of regulatory regions co-bound by both TFs, many of which regulate Notch pathway related genes. Also SOX1B factors (Sox1/2/3) have been previously shown to counteract proneural proteins in gain-of-function experiments in the chick neural tube (Bylund et al., 2003). Thus, the intertwining of transcriptional networks regulating neural progenitor maintenance and differentiation may be a recurrent feature to be explored in future studies.

One immediate example is the Notch pathway. Within the large number of Ascl1 targets identified in ventral telencephalon, the Rbpj consensus binding sequence is enriched at the vicinity of Ascl1 binding sites, specifically in targets that promote proliferation (Castro et al., 2011). Previous studies of neurogenesis in Drosophila provide important clues on how the two factors may interact at the molecular level (Nellesen et al., 1999; Cave et al., 2005). In co-bound regulatory regions with a specific cis-architecture, efficient transactivation is only achieved upon the simultaneous activation of both proneural and Notch pathways. A similar synergy between Ascll and Rbpj can be observed in transcriptional assays in murine cells (Cave et al., 2005), although it remains to be shown whether such interaction does take place in gene regulatory regions. RG cells can be distinguished from intermediate progenitors in ventral telencephalon based on their high levels of canonical Notch signaling 
(Mizutani et al., 2007), thus in principle such a mechanism could be used to differentially activate Ascll targets in the two types of progenitors.

\section{PERSPECTIVE}

Proneural TFs such as Ascll have been seen as master regulators of the neuronal lineage that play an important regulatory role at the onset of differentiation. Recent findings have uncovered a far more complex picture in which Ascll plays sequential functions in proliferating and differentiating neural stem/progenitor cells, with the concomitant regulation of distinct target genes. How sub-sets of the Ascll transcriptional program are differentially activated along the neuronal lineage is poorly understood, and will certainly result from the combination of distinct mechanistic determinants. This important question will surely remain a subject of intense research for the foreseeable future.

\section{ACKNOWLEDGMENTS}

We thank A. Raposo for critically reading this manuscript. Our work is supported by grants PTDC/SAU-BID/117418/2010 and PTDC/NEU-NMC/0315/2012 from FCT and a Marie Curie CIG to DSC. FFV and DSC are recipients of SFRH/BD/51178/2010 and IF/00413/2012 from FCT.

\section{REFERENCES}

Ali, F. R., Cheng, K., Kirwan, P., Metcalfe, S., Livesey, F. J., Barker, R. A., et al. (2014). The phosphorylation status of Ascll is a key determinant of neuronal differentiation and maturation in vivo and in vitro. Development 141, 22162224. doi: 10.1242/dev.106377

Ali, F., Hindley, C., McDowell, G., Deibler, R., Jones, A., Kirschner, M., et al. (2011). Cell cycle-regulated multi-site phosphorylation of neurogenin 2 coordinates cell cycling with differentiation during neurogenesis. Development 138, 4267-4277. doi: 10.1242/dev.067900

Berninger, B., Costa, M. R., Koch, U., Schroeder, T., Sutor, B., Grothe, B., et al. (2007a). Functional properties of neurons derived from in vitro reprogrammed postnatal astroglia. J. Neurosci. 27, 8654-8664. doi: 10.1523/jneurosci.1615-07. 2007

Berninger, B., Guillemot, F., and Götz, M. (2007b). Directing neurotransmitter identity of neurones derived from expanded adult neural stem cells. Eur. J. Neurosci. 25, 2581-2590. doi: 10.1111/j.1460-9568.2007.05509.x

Bertrand, N., Castro, D. S., and Guillemot, F. (2002). Proneural genes and the specification of neural cell types. Nat. Rev. Neurosci. 3, 517-530. doi: 10. 1038/nrn874

Blackwell, T. K., and Weintraub, H. (1990). Preferences of MyoD and E2A protein complexes revealed by binding site selection. Science 250, 1104-1110. doi: 10 . $1126 /$ science. 2174572

Borromeo, M. D., Meredith, D. M., Castro, D. S., Chang, J. C., Tung, K.-C., Guillemot, F., et al. (2014). A transcription factor network specifying inhibitory versus excitatory neurons in the dorsal spinal cord. Development 141, 28032812. doi: 10.1242/dev.105866

Bylund, M., Andersson, E., Novitch, B. G., and Muhr, J. (2003). Vertebrate neurogenesis is counteracted by Sox1-3 activity. Nat. Neurosci. 6, 1162-1168. doi: 10 . 1038/nn1131

Casarosa, S., Fode, C., and Guillemot, F. (1999). Mash1 regulates neurogenesis in the ventral telencephalon. Development 126, 525-534.

Castella, P., Sawai, S., Nakao, K., Wagner, J. A., and Caudy, M. (2000). HES-1 repression of differentiation and proliferation in PC12 cells: role for the helix 3-helix 4 domain in transcription repression. Mol. Cell. Biol. 20, 6170-6183. doi: $10.1128 / \mathrm{mcb} \cdot 20.16 .6170-6183.2000$

Castro, D. S., Martynoga, B., Parras, C., Ramesh, V., Pacary, E., Johnston, C., et al. (2011). A novel function of the proneural factor Ascll in progenitor proliferation identified by genome-wide characterization of its targets. Genes Dev. 25, 930-945. doi: 10.1101/gad.627811
Castro, D. S., Skowronska-Krawczyk, D., Armant, O., Donaldson, I. J., Parras, C., Hunt, C., et al. (2006). Proneural bHLH and Brn proteins coregulate a neurogenic program through cooperative binding to a conserved DNA motif. Dev. Cell 11, 831-844. doi: 10.1016/j.devcel.2006.10.006

Cave, J. W., Loh, F., Surpris, J. W., Xia, L., and Caudy, M. A. (2005). A DNA transcription code for cell-specific gene activation by notch signaling. Curr. Biol. 15, 94-104. doi: 10.1016/j.cub.2004.12.070

Fisher, F., Crouch, D. H., Jayaraman, P. S., Clark, W., Gillespie, D. A., and Goding, C. R. (1993). Transcription activation by Myc and Max: flanking sequences target activation to a subset of CACGTG motifs in vivo. EMBO J. 12, 5075-5082.

Gaudet, J., and Mango, S. E. (2002). Regulation of organogenesis by the Caenorhabditis elegans FoxA protein PHA-4. Science 295, 821-825. doi: 10.1126/science. 1065175

Geoffroy, C. G., Critchley, J. A., Castro, D. S., Ramelli, S., Barraclough, C., Descombes, P., et al. (2009). Engineering of dominant active basic helix-loophelix proteins that are resistant to negative regulation by postnatal central nervous system antineurogenic cues. Stem Cells 27, 847-856. doi: 10.1002/stem. 17

Götz, M., and Huttner, W. B. (2005). The cell biology of neurogenesis. Nat. Rev. Mol. Cell Biol. 6, 777-788. doi: 10.1038/nrm1739

Hartman, J., Müller, P., Foster, J. S., Wimalasena, J., Gustafsson, J.-A., and Ström, A. (2004). HES-1 inhibits 17beta-estradiol and heregulin-betal-mediated upregulation of E2F-1. Oncogene 23, 8826-8833. doi: 10.1038/sj.onc.1208139

Haubensak, W., Attardo, A., Denk, W., and Huttner, W. B. (2004). Neurons arise in the basal neuroepithelium of the early mammalian telencephalon: a major site of neurogenesis. Proc. Natl. Acad. Sci. U S A 101, 3196-3201. doi: 10.1073/pnas. 0308600100

Henke, R. M., Meredith, D. M., Borromeo, M. D., Savage, T. K., and Johnson, J. E. (2009). Ascl1 and Neurog2 form novel complexes and regulate Delta-like3 (Dll3) expression in the neural tube. Dev. Biol. 328, 529-540. doi: 10.1016/j.ydbio.2009. 01.007

Hirata, H., Yoshiura, S., Ohtsuka, T., Bessho, Y., Harada, T., Yoshikawa, K., et al. (2002). Oscillatory expression of the bHLH factor Hes 1 regulated by a negative feedback loop. Science 298, 840-843. doi: 10.1126/science. 1074560

Horton, S., Meredith, A., Richardson, J. A., and Johnson, J. E. (1999). Correct coordination of neuronal differentiation events in ventral forebrain requires the bHLH factor MASH1. Mol. Cell. Neurosci. 14, 355-369. doi: 10.1006/mcne.1999. 0791

Imayoshi, I., Isomura, A., Harima, Y., Kawaguchi, K., Kori, H., Miyachi, H., et al. (2013). Oscillatory control of factors determining multipotency and fate in mouse neural progenitors. Science 342, 1203-1208. doi: 10.1126/science. 1242366

Jacob, J., Kong, J., Moore, S., Milton, C., Sasai, N., Gonzalez-Quevedo, R., et al. (2013). Retinoid acid specifies neuronal identity through graded expression of Ascl1. Curr. Biol. 23, 412-418. doi: 10.1016/j.cub.2013.01.046

Kageyama, R., Ohtsuka, T., Hatakeyama, J., and Ohsawa, R. (2005). Roles of bHLH genes in neural stem cell differentiation. Exp. Cell Res. 306, 343-348. doi: 10. 1016/j.yexcr.2005.03.015

Karow, M., Sánchez, R., Schichor, C., Masserdotti, G., Ortega, F., Heinrich, C., et al. (2012). Reprogramming of pericyte-derived cells of the adult human brain into induced neuronal cells. Cell Stem Cell 11, 471-476. doi: 10.1016/j.stem.2012.07. 007

Kobayashi, T., Mizuno, H., Imayoshi, I., Furusawa, C., Shirahige, K., and Kageyama, R. (2009). The cyclic gene Hes1 contributes to diverse differentiation responses of embryonic stem cells. Genes Dev. 23, 1870-1875. doi: 10.1101/gad.1823109

Kriegstein, A., and Alvarez-Buylla, A. (2009). The glial nature of embryonic and adult neural stem cells. Annu. Rev. Neurosci. 32, 149-184. doi: 10.1146/annurev. neuro.051508.135600

Li, S., Mattar, P., Dixit, R., Lawn, S. O., Wilkinson, G., Kinch, C., et al. (2014). RAS/ERK signaling controls proneural genetic programs in cortical development and gliomagenesis. J. Neurosci. 34, 2169-2190. doi: 10.1523/jneurosci. 4077-13.2014

Louvi, A., and Artavanis-Tsakonas, S. (2006). Notch signalling in vertebrate neural development. Nat. Rev. Neurosci. 7, 93-102. doi: 10.1038/nrn 1847

Mangan, S., and Alon, U. (2003). Structure and function of the feed-forward loop network motif. Proc. Natl. Acad. Sci. US A 100, 11980-11985. doi: 10.1073/pnas. 2133841100

Marin, O., Anderson, S. A., and Rubenstein, J. L. (2000). Origin and molecular specification of striatal interneurons. J. Neurosci. 20, 6063-6076. 
Masamizu, Y., Ohtsuka, T., Takashima, Y., Nagahara, H., Takenaka, Y., Yoshikawa, K., et al. (2006). Real-time imaging of the somite segmentation clock: revelation of unstable oscillators in the individual presomitic mesoderm cells. Proc. Natl. Acad. Sci. U S A 103, 1313-1318. doi: 10.1073/pnas.0508658103

Mengel, B., Hunziker, A., Pedersen, L., Trusina, A., Jensen, M. H., and Krishna, S. (2010). Modeling oscillatory control in NF- $\kappa$ B, p53 and Wnt signaling. Curr. Opin. Genet. Dev. 20, 656-664. doi: 10.1016/j.gde.2010.08.008

Miyata, T., Kawaguchi, A., Saito, K., Kawano, M., Muto, T., and Ogawa, M. (2004). Asymmetric production of surface-dividing and non-surface-dividing cortical progenitor cells. Development 131, 3133-3145. doi: 10.1242/dev. 01173

Mizutani, K., Yoon, K., Dang, L., Tokunaga, A., and Gaiano, N. (2007). Differential notch signalling distinguishes neural stem cells from intermediate progenitors. Nature 449, 351-355. doi: 10.1038/nature06090

Nakada, Y., Hunsaker, T. L., Henke, R. M., and Johnson, J. E. (2004). Distinct domains within Mash1 and Math1 are required for function in neuronal differentiation versus neuronal cell-type specification. Development 131, 13191330. doi: 10.1242/dev.01008

Nellesen, D. T., Lai, E. C., and Posakony, J. W. (1999). Responsiveness of enhancer of split complex genes to common transcriptional activators. Dev. Biol. 53, 33-53.

Noctor, S. C., Martínez-Cerdeño, V., Ivic, L., and Kriegstein, A. R. (2004). Cortical neurons arise in symmetric and asymmetric division zones and migrate through specific phases. Nat. Neurosci. 7, 136-144. doi: 10.1038/nn1172

Oishi, K., Watatani, K., Itoh, Y., Okano, H., Guillemot, F., Nakajima, K., et al. (2009). Selective induction of neocortical GABAergic neurons by the PDK1-Akt pathway through activation of Mash1. Proc. Natl. Acad. Sci. U S A 106, 1306413069. doi: 10.1073/pnas.0808400106

Pilz, G.-A., Shitamukai, A., Reillo, I., Pacary, E., Schwausch, J., Stahl, R., et al. (2013). Amplification of progenitors in the mammalian telencephalon includes a new radial glial cell type. Nat. Commun. 4:2125. doi: 10.1038/ncomms3125

Pina, C., Fugazza, C., Tipping, A. J., Brown, J., Soneji, S., Teles, J., et al. (2012). Inferring rules of lineage commitment in haematopoiesis. Nat. Cell. Biol. 14, 287-294. doi: 10.1038/ncb2442

Renault, V. M., Rafalski, V. A., Morgan, A. A., Salih, D. A. M., Brett, J. O., Webb, A. E., et al. (2009). FoxO3 regulates neural stem cell homeostasis. Cell Stem Cell 5, 527-539. doi: 10.1016/j.stem.2009.09.014

Sang, L., Coller, H. A., and Roberts, J. M. (2008). Control of the reversibility of cellular quiescence by the transcriptional repressor HES1. Science 321, 10951100. doi: 10.1126/science. 1155998

Seo, S., Richardson, G. A., and Kroll, K. L. (2005). The SWI/SNF chromatin remodeling protein Brg1 is required for vertebrate neurogenesis and mediates transactivation of Ngn and NeuroD. Development 132, 105-115. doi: 10. 1242/dev.01548

Sequerra, E. B., Costa, M. R., Menezes, J. R. L., and Hedin-Pereira, C. (2013). Adult neural stem cells: plastic or restricted neuronal fates?. Development 140, 33033309. doi: 10.1242/dev.093096
Shen-Orr, S. S., Milo, R., Mangan, S., and Alon, U. (2002). Network motifs in the transcriptional regulation network of Escherichia coli. Nat. Genet. 31, 64-68. doi: $10.1038 /$ ng 881

Shimojo, H., Ohtsuka, T., and Kageyama, R. (2008). Oscillations in notch signaling regulate maintenance of neural progenitors. Neuron 58, 52-64. doi: 10.1016/j. neuron.2008.02.014

Soufi, A., Donahue, G., and Zaret, K. S. (2012). Facilitators and impediments of the pluripotency reprogramming factors' initial engagement with the genome. Cell 151, 994-1004. doi: 10.1016/j.cell.2012.09.045

Sriuranpong, V., Borges, M. W., Christopher, L., Nakakura, E. K., Watkins, D. N., Christine, M., et al. (2002). Notch signaling induces rapid degradation of achaete-scute homolog 1 notch signaling induces rapid degradation of achaetescute homolog 1. Mol. Cell. Biol. 22, 3129-3139. doi: 10.1128/mcb.22.9.31293139.2002

Vierbuchen, T., Ostermeier, A., Pang, Z. P., Kokubu, Y., Südhof, T. C., and Wernig, M. (2010). Direct conversion of fibroblasts to functional neurons by defined factors. Nature 463, 1035-1041. doi: 10.1038/nature08797

Viñals, F., Reiriz, J., Ambrosio, S., Bartrons, R., Rosa, J. L., and Ventura, F. (2004). BMP-2 decreases Mash1 stability by increasing Id1 expression. EMBO J. 23, 3527-3537. doi: 10.1038/sj.emboj.7600360

Wapinski, O. L., Vierbuchen, T., Qu, K., Lee, Q. Y., Chanda, S., Fuentes, D. R., et al. (2013). Hierarchical mechanisms for direct reprogramming of fibroblasts to neurons. Cell 155, 621-635. doi: 10.1016/j.cell.2013.09.028

Webb, A. E., Pollina, E. A., Vierbuchen, T., Urbán, N., Ucar, D., Leeman, D. S., et al. (2013). FOXO3 shares common targets with ASCL1 genome-wide and inhibits ASCL1-dependent neurogenesis. Cell Rep. 4, 477-491. doi: 10.1016/j. celrep.2013.06.035

Wilkinson, G., Dennis, D., and Schuurmans, C. (2013). Proneural genes in neocortical development. Neuroscience 253, 256-273. doi: 10.1016/j.neuroscience.2013. 08.029

Conflict of Interest Statement: The authors declare that the research was conducted in the absence of any commercial or financial relationships that could be construed as a potential conflict of interest.

Received: 30 August 2014; accepted: 12 November 2014; published online: 02 December 2014.

Citation: Vasconcelos FF and Castro DS (2014) Transcriptional control of vertebrate neurogenesis by the proneural factor Ascll. Front. Cell. Neurosci. 8:412. doi: 10.3389/fncel.2014.00412

This article was submitted to the journal Frontiers in Cellular Neuroscience.

Copyright (C) 2014 Vasconcelos and Castro. This is an open-access article distributed under the terms of the Creative Commons Attribution License (CC BY). The use, distribution and reproduction in other forums is permitted, provided the original author(s) or licensor are credited and that the original publication in this journal is cited, in accordance with accepted academic practice. No use, distribution or reproduction is permitted which does not comply with these terms. 